naukowych. Konferencji Miedzynarodowej Naukowo-Praktycznej "Inzynieria i technologia. Teoretyczne i praktyczne aspekty rozwoju wspolczesnej nauki" (30.03.2017-31.03.2017) - Warszawa: Wydawca: Sp. z o.o. "Diamond trading tour. 2017. - C. 42-46.

2. Gutsul O. V. Skin-effect of fluid in capillaries / O. V. Gutsul, V. Z. Slobodian. // SWorld Journal. - 2016. - №11, vol. 10 - C. 44-47.

Статья отправлена: 5.06.2017 г.

(C) Гуцул О.В., Слободян В.3.

\title{
ЦИТ: иа117-082
}

DOI: 10.21893/2415-7538.2016-06-5-082

УДК 517.9

\section{ОПТИМАЛЬНЫЙ УГОЛ БРОСАНИЯ ТЕЛ \\ В ПОЛЕ ТЯЖЕСТИ ЗЕМЛИ С УЧЕТОМ ЕЕ ВРАЩЕНИЯ}

Окулов С.А., Демина Е.Л.

Уральский федеральный университет,

Нижнетагильский технологический институт (филиал),

г. Нижний Тагил, Свердловская область

Okulov S.A., Demina E.L.

\section{OPTIMUM ANGEL BODY TOSSING IN THE EARTH GRAVITY} FIELD CONSIDERING ITS ROTATION

Ural Federal University, Nizhny Tagil Technological Institute (branch), Nizhny Tagil, Sverdlovskaya Region

Аннотачия: В работе операчионным методом получено точное решение задачи о влиянии вращения Земли на движение тела при ненулевых начальных условиях. Исследовано влияние вращения Земли на оптимальный угол бросания.

Ключевые слова: Движение в поле тяжести; уравнения движения; оптимальный угол бросания.

Abstract. In this paper by operation method the exact solution of a task about influence of the Earth rotation is received on body movement upon non-zero initial conditions. There has been studied the Earth rotation impact on the optimum angle tossing.

Key words : Motion in a gravitational field; equations of motion; optimal angle tossing.

Изучим влияние вращения Земли на движение тел в поле ее тяжести, задаваемого следующей системой дифференциальных уравнений [1]:

$$
\left\{\begin{array} { l } 
{ \ddot { x } = 2 \omega ( \dot { z } \operatorname { c o s } \varphi + \dot { y } \operatorname { s i n } \varphi ) , } \\
{ \ddot { y } = - 2 \omega \dot { x } \operatorname { s i n } \varphi , } \\
{ \ddot { z } = - g - 2 \omega \dot { x } \operatorname { c o s } \varphi , }
\end{array} \text { при начальных условиях } \left\{\begin{array}{l}
t=0, \\
x(0)=0, \dot{x}(0)=u, \\
y(0)=0, \dot{y}(0)=v, \\
z(0)=0, \dot{z}(0)=w,
\end{array}\right.\right.
$$

где угол $\varphi$ - географическая широта в данной точке земной поверхности, 
$a=\cos \varphi, \quad b=\sin \varphi, \omega=\frac{2 \pi}{26 \cdot 60 \cdot 60} \approx 0,000073 c^{-1}$-угловая скорость вращения Земли вокруг своей оси; $u, v, w-$ компоненты начальной скорости $V_{0}$.

Ось $z$ направлена по вертикали вверх, ось $x$ - по касательной к окружности радиуса $h$, а ось $y$ - в плоскости меридиана.

При расчетах будем использовать аппарат операционного исчисления [2], заметно упрощающий математические выкладки.

Для решения приведенной системы воспользуемся преобразованием Лапласа:

$$
f(t)=p F(p), f^{\prime}(t)=p F(p)-f(0), f^{n}(t)=p^{n} F(p)-f(0)-f^{\prime}(0) .
$$

Получим следующие изображения:

$$
\begin{aligned}
& x(t) \stackrel{\bullet}{\longleftarrow} X(p), \quad \dot{x}(t) \stackrel{\bullet}{\longleftarrow} p(p), \\
& \ddot{x}(t) \stackrel{\bullet}{\longleftarrow} p^{2} X(p)-u ; \\
& y(t) \stackrel{\bullet}{\longleftarrow} Y(p), \quad \dot{y}(t) \stackrel{\bullet}{\longleftarrow} p(p), \\
& \ddot{y}(t) \stackrel{\bullet}{\longleftarrow} p^{2} Y(p)-v ; \\
& z(t) \stackrel{\bullet}{\longleftarrow} Z(p), \quad \dot{z}(t) \stackrel{\bullet}{\longleftarrow} Z(p), \\
& \ddot{z}(t) \stackrel{\bullet}{\longleftarrow} p^{2} Z(p)-w ; \\
& g \leftarrow \frac{g}{p} \text {. }
\end{aligned}
$$

Применим преобразование Лапласа к каждому из уравнений системы:

$$
\left\{\begin{array}{l}
p^{2} X(p)-2 \omega p b Y(p)-2 \omega a p Z(p)=u, \\
2 \omega b p X(p)+p^{2} Y(p)=v \\
2 \operatorname{\omega ap} X(p)+p^{2} Z(p)=w-\frac{g}{p}
\end{array}\right.
$$

Решая полученную систему уравнений, для изображения имеем:

$$
\left\{\begin{array}{l}
X(p)=-\frac{a g}{2 \omega}\left(\frac{1}{p^{2}}-\frac{1}{2 \omega} \frac{2 \omega}{p^{2}+4 \omega^{2}}\right)+\frac{u}{2 \omega} \cdot \frac{2 \omega}{p^{2}+4 \omega^{2}}+\frac{a w+b v}{2 \omega}\left(\frac{1}{p}-\frac{p}{p^{2}+4 \omega^{2}}\right) \\
Y(p)=4 a b g \omega^{2}\left(\frac{2}{2 p^{3}} \frac{1}{4 \omega^{2}}-\frac{1}{16 \omega^{4} p}+\frac{p}{16 \omega^{4}\left(p^{2}+4 \omega^{2}\right)}\right)+\frac{v}{p^{2}+4 \omega^{2}}- \\
-2 \omega b u\left(\frac{1}{4 \omega^{2}} \frac{1}{p}-\frac{1}{4 \omega^{2}} \frac{p}{p^{2}+4 \omega^{2}}\right)+4 \omega^{2}\left(a^{2} v-a b w\right)\left(\frac{1}{4 \omega^{2}} \frac{1}{p^{2}}-\frac{1}{4 \omega^{2}} \frac{1}{p^{2}+4 \omega^{2}}\right) \\
Z(p)=-4 \omega^{2} b^{2} g\left(\frac{1}{4 \omega^{2}} \frac{1}{p^{3}}-\frac{1}{16 \omega^{4}} \cdot \frac{1}{p}+\frac{1}{16 \omega^{4}} \cdot \frac{p}{p^{2}+4 \omega^{2}}\right)+\frac{w}{2 \omega} \cdot \frac{2 \omega}{p^{2}+4 \omega^{2}}- \\
-\frac{2 \omega a u+g}{4 \omega^{2}}\left(\frac{1}{p}-\frac{p}{p^{2}+4 \omega^{2}}\right)+\left(w b^{2}-a b v\right) \cdot\left(\frac{1}{p^{2}}-\frac{1}{p^{2}+4 \omega^{2}}\right) .
\end{array}\right.
$$

Тогда уравнения движения тела имеют вид: 


$$
\left\{\begin{array}{l}
x(t)=\frac{a g}{2 \omega}\left(\frac{\sin 2 \omega t}{2 \omega}-t\right)+\frac{u}{2 \omega} \sin 2 \omega t+\frac{b v}{\omega} \sin ^{2} \omega t+\frac{a w}{\omega} \sin ^{2} \omega t \\
y(t)=\frac{g a b}{2 \omega^{2}}\left(\omega^{2} t^{2}-\sin ^{2} \omega t\right)-\frac{b u}{\omega} \sin ^{2} \omega t+\frac{v}{2 \omega}\left(b^{2} \sin 2 \omega t+2 a^{2} \omega t\right)- \\
-\frac{a b w}{2 \omega}(2 \omega t-\sin 2 \omega t), \\
z(t)=-\frac{g}{2 \omega^{2}}\left(b^{2} \omega^{2} t^{2}+a^{2} \sin ^{2} \omega t\right)-\frac{a u}{\omega} \sin ^{2} \omega t-\frac{a b v}{2 \omega}(2 \omega t-\sin 2 \omega t)+ \\
+\frac{w}{2 \omega}\left(2 \omega t b^{2}+a^{2} \sin 2 \omega t\right) .
\end{array}\right.
$$

Полученные уравнения позволяют оценить влияние вращения Земли на величину оптимального угла бросания тела в поле ее тяжести.

Проведем расчеты для тела, брошенного с запада на восток в северном полушарии на широте города Нижний Тагил $\left(\varphi=58^{0}\right)$.

Результаты расчетов для различных начальных скоростей представлены на рис.1.

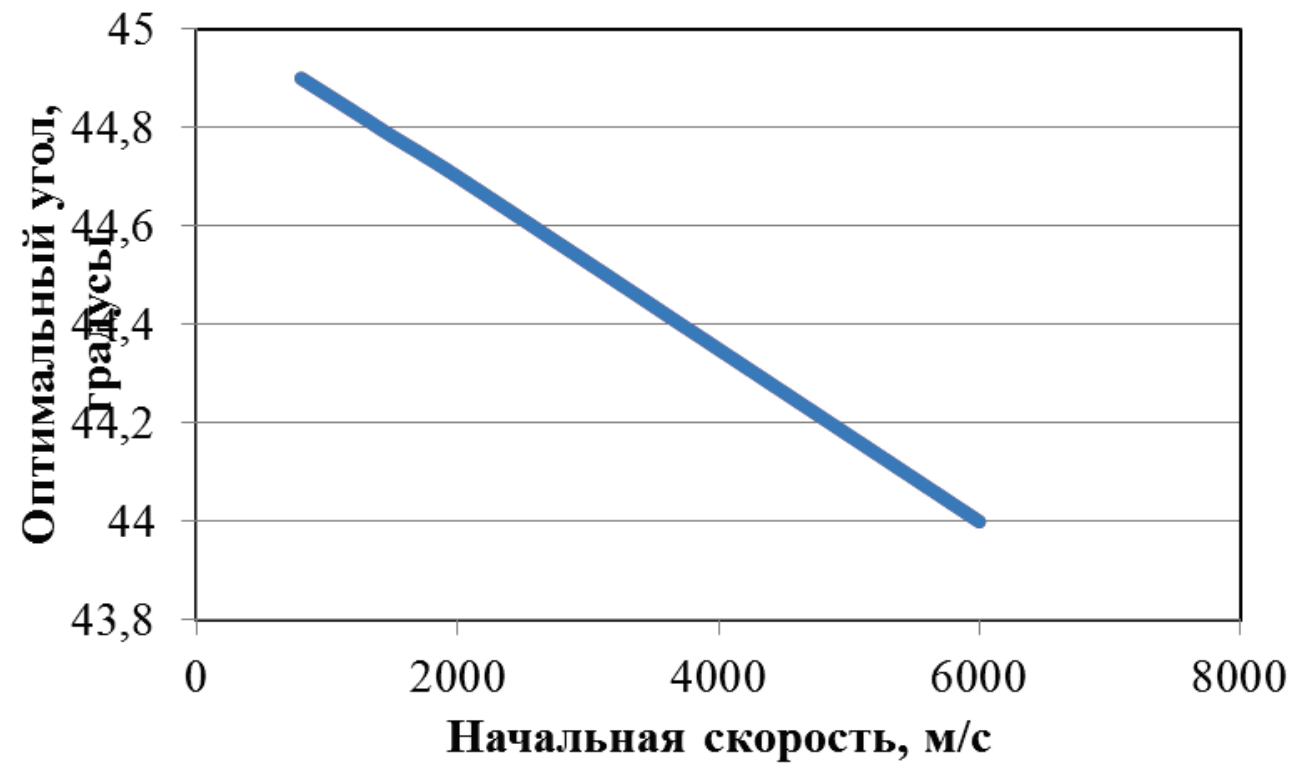

Рис.1. Зависимость оптимального угла от начальной скорости тела

Таким образом, расчеты показывают, что вращение Земли уменьшает оптимальный угол стрельбы, причем чем выше начальная скорость тела, тем больше отклонение величины оптимального угла стрельбы от $45^{\circ}$.

Полученные данные хорошо согласуются с результатами расчетов оптимального угла в рамках эллиптической теории движения, приведенными в работе [3].

\section{Литература}

1. Дмитриевский А.А., Лысенко Л.Н. Внешняя баллистика: Учебник для студентов вузов. - 4-е изд., перераб. и доп. - М.: Машиностроение, 2005. 
2. Пантелеев А. В. Теория функции комплексного переменного и операционного исчисления в примерах и задачах / А. В. Пантелеев, А. С. Якимова. - М. : МАИ, 1998.

3. Лахтин Л.М. Свободное движение в поле земного сфероида - М. : Физматгиз, 1963.

Статья отправлена: 11.06.2017 г. (C) Окулов С.А., Демина Е.Л. 